\title{
Interdisciplinary Management of Gummy Smile and Fluorosis - A Case Report
}

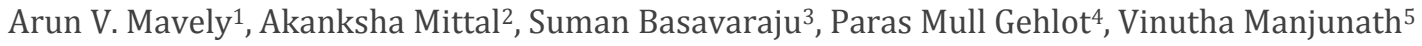 \\ 1, 3 Department of Periodontics, JSS Dental College and Hospital, JSS Academy of Higher Education \\ and Research, Sri Shivarathreeshwara Nagar, Mysuru, Karnataka, India. 2, 4, 5 Department of \\ Conservative Dentistry and Endodontics, JSS Dental College and Hospital, JSS Academy of Higher \\ Education and Research, Sri Shivarathreeshwara Nagar, Mysuru, Karnataka, India.
}

\section{INTRODUCTION}

An unpleasant smile can have a psychosocial effect leading to lack of self-esteem and a challenge in social relations. A perfect smile is a balance among 3 parameters: the white (teeth), the pink (gum) and the lips. A predictable smile correction warrants a multidisciplinary approach and good treatment planning. We planned for a minimally invasive interdisciplinary treatment approach for the restoration of a gummy smile with dental fluorosis in a young female patient.

An interdisciplinary management of smile correction is crucial for successful results. Lasers for soft tissue management and minimal tooth preparation for direct composite veneer restorations being minimally invasive periodontal and restorative approaches were the key to success with exceptional patient satisfaction.

Minimally invasive procedures in interdisciplinary dentistry should be embraced in the management of procedures requiring correction of both hard and soft tissues, for successful outcomes. Pre-treatment mock-up can build patients' confidence in dentistry and are useful tools for planning treatments with a predictable outcome.

A beautiful smile is the fruit of a coordinated and balanced interplay between teeth, gingiva and lips. A compromise in any of these cornerstones can impair the beauty of a smile. Thorough knowledge and understanding about the various elements involved and their harmonious synchronisation makes smile makeovers a challenging task. One such challenge is the treatment of gummy smile.

A gummy smile is a clinical condition that has gained tremendous focus and attention recently. Gummy smile is the excessive gingival display when a person smiles. It has been defined as a nonpathological condition causing aesthetic disharmony in which more than $3 \mathrm{~mm}$ of gingival tissue is exposed while smiling. ${ }^{1}$ Aetiology of a gummy smile can be multifactorial and the main factors being altered passive eruption (APE), vertical maxillary excess and upper lip hypermobility. The diagnosis must be accurate to deliver the appropriate line of treatment. Based on the complexity of the underlying aetiological factors involved, the treatment modalities also change from conservative approaches like aesthetic restorations and orthodontic tooth movements to periodontal and maxillofacial surgical procedures. ${ }^{2}$

Altered passive eruption (APE) also knows as "impaired passive eruption" is a clinical condition characterised by the coronally positioned marginal gingiva in relation to the cementoenamel junction (CEJ). Passive eruption is a physiological phase involved in the process of tooth eruption. As the teeth meet the occlusal plane, the soft tissue covering the teeth migrates apically till the CEJ is reached. Retardation in this process causes failure of the gingiva to migrate apically, resulting in short clinical crowns and excessive soft tissue exposure while smiling. This is visualised as a gummy smile.

Moreover, the failure of the marginal gingiva to migrate apically forms pseudopockets that can eventually end up in periodontitis. Hence correction of this malformation is not only aesthetically relevant but also functionally rewarding. Coslet et al., have classified altered passive eruption considering the amount of keratinised gingiva and the distance of the CEJ from the alveolar crest. ${ }^{3}$ Accordingly, the treatment has also been proposed that extends from gingivectomy to flap surgeries which may or may not involve extensive osseous reductions based on the corresponding clinical scenario. Armamentarium used can also vary from conventional scalpels to the latest advancements like lasers and peizosurgeries.
Corresponding Author: Dr. Akanksha Mittal, Room No. 7, JSS Dental College and Hospital, JSS Academy of Higher Education and Research, Sri. Shivarathreeshwara Nagara. Mysore 570015, Karnataka, India. E-mail: gunjan.mittal30@gmail.com

DOI: $10.14260 / \mathrm{jemds} / 2021 / 137$

How to Cite This Article:

Mavely AV, Mittal A, Basavaraju S, et al. Interdisciplinary management of gummy smile and fluorosis - a case report. J Evolution Med Dent Sci 2021;10(09):645648, DOI: $10.14260 /$ jemds/2021/137

Submission 06-11-2020,

Peer Review 06-01-2021,

Acceptance 12-01-2021,

Published 01-03-2021.

Copyright (c) 2021 Arun Mavely et al. This is an open access article distributed under Creative Commons Attribution License [Attribution 4.0 International (CC BY 4.0)] 
Along with soft tissue management, aesthetically acceptable teeth play an intricate role in smile corrections. A healthy interrelationship between teeth and periodontium maximises long term stability of treatment outcomes. Restoring teeth with structural and morphological defects like fluorosis demands more comprehensive measures. Fluorosis is a tooth malformation with the characteristic feature of surface hypomineralisation and subsurface hypomineralisation. Fluoride content in drinking water exceeding $0.5-1.5 \mathrm{mg} / \mathrm{l}$ is the aetiology behind this structural alteration of the enamel. Fluoride interferes with the metabolism in ameloblasts causing defective matrix formation and disturbances in calcification. Clinical presentation of the enamel can vary depending on the severity, frequency, and duration of exposure to fluoride. Discoloration may vary from white to yellow to dark brown often accompanied by the associated structural losses like surface pitting defects and attritions. ${ }^{4}$ The diagnosis for dental fluorosis can be often challenging for clinicians because the clinical manifestations may resemble incipient carious lesions and non-fluorotic defects like amelogenesis imperfecta. Thylstrup and Fejerskov index has classified dental fluorosis based on which the clinical management differs. ${ }^{5}$ Methods of treatment can vary from microabrasion and bleaching for mild cases to veneering and crowns in moderate and severe cases.

Interdisciplinary dentistry is a combined and interdependent effort among the different dental specialties. Interdisciplinary assessment of clinical scenarios has become extremely helpful for accurate diagnosis and effective treatments. As the aetiology can be multifactorial, smile corrections also require multidisciplinary approaches for long term and sustainable results. In this case report, we discuss an interdisciplinary treatment plan to correct the gummy smile of a young female patient with fluorosis using minimally invasive approaches involving aesthetic crown lengthening with a diode laser and direct composite veneering technique.

\section{PRESENTATION OF CASE}

A 26-year-old female patient reported to the Department of Periodontology with a chief complaint of an unaesthetic smile (Fig. 1a). The patient's medical history was non-contributing. On intraoral examination, the patient had excessive gingival display accompanied with maxillary short clinical crowns with pseudo pockets which were diagnostic features of gummy smile due to altered passive eruption (APE), enamel had pitted surface with an opaque appearance that was suggestive of moderate enamel fluorosis (TFI score 5), and Ellis Class II fracture of upper left central and lateral incisors (\# 21, \#22). On radiographic evaluation, mild interdental bone loss was noted. The pulp vitality test was positive for all the upper incisors.

\section{DISCUSSION OF MANAGEMENT}

After complete clinical, radiographic evaluation and investigations, a comprehensive treatment approach was planned. The treatment approaches consisted of aesthetic crown lengthening using a diode laser $(808 \mathrm{~nm})$ followed by direct composite veneering.
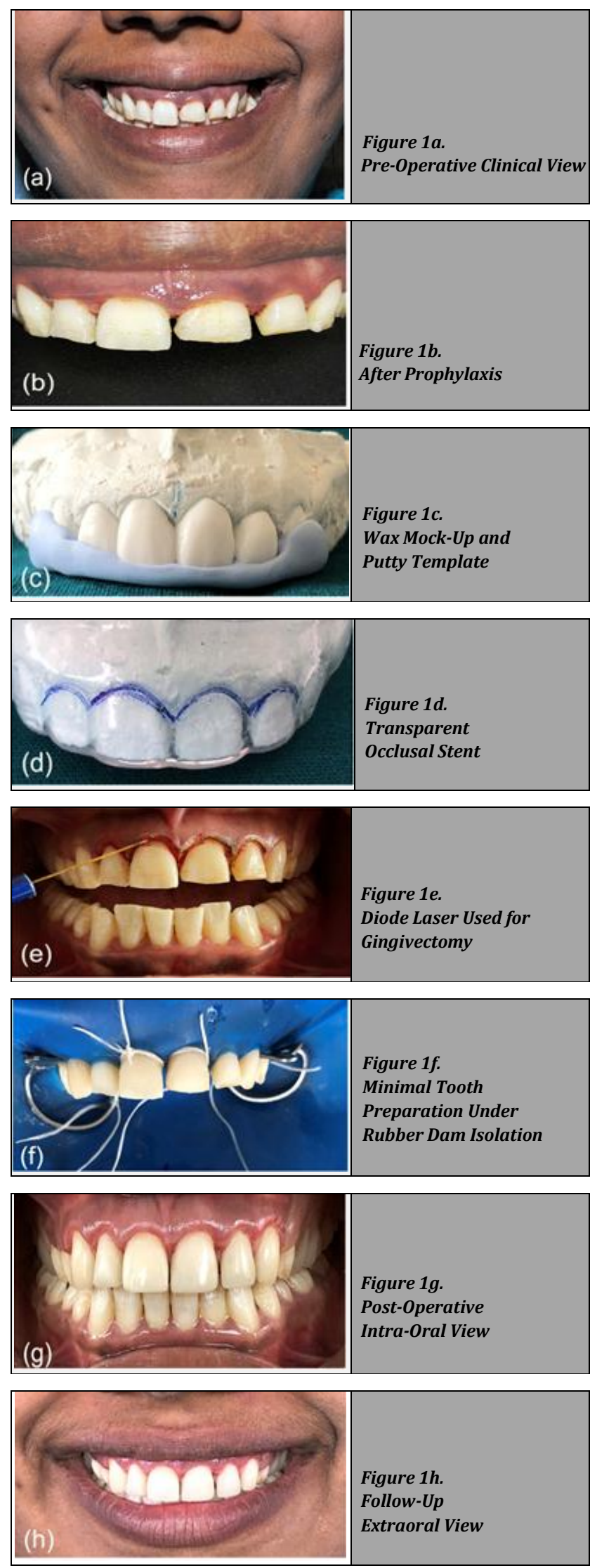

After obtaining informed consent from the patient, the procedures were initiated. The steps followed were - 


\section{Step 1 - Oral Prophylaxis}

- $\quad$ Supra-gingival, subgingival scaling and root planning (Fig. 1b). The extent of gingivectomy was estimated by measuring the distance from the marginal gingiva to the crest of the alveolar bone with the help of a trans-gingival probing method using a UNC-15 probe.

\section{Step 2 - Study Model with Mock Gingivectomy}

- The upper and lower impressions were made with alginate (DPI, India) and models poured. On the maxillary models, a mock gingivectomy procedure was performed with a straight fissure bur.

- The clinical crown proportions of at least $80 \%$ (length: width) were maintained in all upper incisors.

\section{Step 3 - Fabrication of the Wax Mock-Up}

- A wax-up was performed on upper four incisors and an index was made using putty consistency addition silicone elastomeric material (Aquasil. Dentsply. India) (Fig. 1c).

\section{Step 4 - Fabrication of a Transparent Occlusal Stent (Splint)}

- to aid in predictable placement and achieving ideal scalloping of the final gingival margin (Fig. 1d).

\section{Step 5 - Aesthetic Crown Lengthening (Gingivectomy)}

- Under local anaesthesia (2 \% lignocaine hydrochloride), the portion of the gingiva to be excised was marked after placing the transparent stent intraorally.

- The gingivectomy was performed with Sunny Diode laser $(808 \mathrm{~nm})$ (Sunny Optoelectronic Technology Co. Ltd. Shanghai, China) (Fig. 1e) on continuous mode under 4-watt power and the excised portion of the gingiva was removed with a universal curette. The site was irrigated with $0.9 \%$ normal saline. The patient was discharged after prescribing analgesics and chlorhexidine mouth wash. The patient was recalled after 4 weeks to assess the healing and was found to be satisfactory.

\section{Step 6 - Direct Composite Veneer Build-Up}

- On a subsequent visit, shade matching was done using VITA shade guide (dentin-B2, and enamel- A2) and rubber dam placed.

- Minimal tooth preparation for partial veneer was done using a long taper bur (847 - 014 / Coarse Grit-Medicept Dental, UK), with a chamfer finish line on teeth \# 12, 11, 21, 22 (Fig. 1f).

- $\quad$ The teeth were acid etched using $37 \%$ phosphoric acid for 30 seconds (additional etching time of 20 seconds was used because teeth were affected with fluorosis), rinsed for 10 seconds and dried. A bonding agent (Tetric N-Bond, Ivoclar Vivadent AG, Liechtenstein) was applied on the prepared enamel and light-cured for 20 seconds.
- Using the putty index technique, the teeth were restored in a layering technique with A2 enamel shade and B2 dentin shade to emulate enamel and dentin respectively.

- $\quad$ Finishing and polishing were performed with finishing burs and polishing kit. (super snap mini kit, Shofu. Kyoto, Japan) (Fig. 1g).

- The rubber dam was removed, and occlusal relations were checked. Oral hygiene instructions were given, and the patient was followed up.

- An 18-month follow-up was satisfactory (Fig. 1h).

\section{DISCUSSION}

A harmonious inter-relationship between the teeth and periodontium is the key to the long-term success of periodontal- restorative treatments. Simulation of the spatial arrangement, relation and appearance of natural tissues is necessary for a successful treatment. ${ }^{6}$ Thorough knowledge of the aesthetic zone, that is the maxillary anterior region precedes the aesthetic crown lengthening procedure. A comprehensive clinical assessment of clinical crown length, the height of the keratinised gingiva, anatomical crown length, and the location of the alveolar crest is important. Application of golden proportions along with these criteria should guide the periodontist's treatment approach as it will direct the extent of gingival excision that is required, with or without osseous reduction. ${ }^{7}$

According to Coslet and colleagues' classification for the different forms of altered passive eruption, the present case corresponds to the class 1 subtype a situation where the treatment modality involves the recontouring of the soft tissue only. Hence, gingivectomy procedure was performed. ${ }^{3}$ The outcome expected was to attain positive osseous and soft tissue contour which ensures that the proposed restorative margin is a minimum of $2 \mathrm{~mm}$ from the marginal bone which is to respect biologic width. The biological width as defined by Gargiulo et al. is "the dimension of the soft tissue, which is attached to the portion of the tooth coronal to the crest of the alveolar bone". The sum of the epithelial $(0.97 \mathrm{~mm})$ and connective tissue measurements $(1.07 \mathrm{~mm})$ makes up the biological width $(2.04 \mathrm{~mm}) .{ }^{8}$ Violation of biologic width results in gingival inflammation around the restoration, bleeding on probing, pocket formation, gingival recession, localised gingival hyperplasia and bone loss. ${ }^{9}$

A diode laser is ideal for soft tissue management as it is absorbed by pigments like melanin and haemoglobin. Moreover, gingivectomy with diode laser is preferred over scalpel because of the several advantages like precision, less time-consumption, minimal invasiveness and comfortable to the patient due to minimum pain. ${ }^{10}$ Further laser promotes the reestablishment of connective tissue attachment and helps in bacterial decontamination. The few disadvantages associated with lasers are occasional tissue necrosis and lateral heat damage. However, in the present case, the healing was uneventful.

Due to advancements in resin bonded composites, their indications and clinical applications have increased fourfold. This transformation may be attributed to the improvements in the physical, mechanical, optical and biological attributes like load resistance, durability, aesthetics, colour stability and 
predictability. These qualities allow for better tooth structure conservation. ${ }^{11}$

Direct veneering employs the application of composite resin material directly on prepared tooth surfaces. Minimal tooth preparation, cost-effective compared with indirect techniques, treatment reversibility and non-requirement of an additional adhesive cementing system and single visit are a few advantages of this technique. Moreover, the polishing of direct veneers is easier and any chipping or fracture of the restoration can be repaired intraorally. Also, marginal adaptation is better when compared with indirect veneer restorations. One of the challenges in the present case was bonding to the fluorosed enamel. ${ }^{12}$ This was addressed by etching for a longer time and keeping the preparation in enamel. Drawbacks of direct laminate veneers include low wear and fracture resistance, and discoloration over a period of time. However, in the present case, the anterior occlusion was favorable and longevity was predictable.

\section{CONCLUSIONS}

Well planned multidisciplinary treatment approaches for the management of compromised smile offers less invasive and cost-effective treatment options for the patients. This article reports successful smile rehabilitation using a minimally invasive periodontal-restorative approach to correct excessive gingival display by laser gingivectomy and restore anterior teeth with fluorosis by direct composite veneers.

Financial or other competing interests: None.

Disclosure forms provided by the authors are available with the full text of this article at jemds.com.

\section{REFERENCES}

[1] Kokich VG. Esthetics: the orthodontic-periodontic restorative connection. Semin Orthod 1996;2(1):21-30.

[2] Silberberg N, Goldstein M, Smidt A. Excessive gingival display--etiology, diagnosis and treatment modalities. Quintessence Int 2009;40(10):809-18.

[3] Coslet JG, Vanarsdall R, Weisgold A. Diagnosis and classification of delayed passive eruption of the dentogingival junction in the adult. Alpha Omegan 1977;70(3):24-8.

[4] Fejerskov 0, Larsen MJ, Richards A, et al. Dental tissue effects of fluoride. Adv Dent Res 1994;8(1):15-31.

[5] Thylstrup A, Fejerskov 0. Clinical appearance of dental fluorosis in permanent teeth in relation to histologic changes. Community Dent Oral Epidemiol 1978;6(6):31528.

[6] Dietschi D. Free-hand bonding in the esthetic treatment of anterior teeth: creating the illusion. J Esthet Dent 1997;9(4):156-64.

[7] Allen EP. Use of mucogingival surgical procedures to enhance esthetics. Dent Clin North Am 1988;32(2):30730.

[8] Gargiulo AW, Wentz FM, Orban B. Dimensions and relations of the dentogingival junction in humans. J Periodontol 1961;32(3):261-7.

[9] Jorgic-Srdjak K, Plancak D, Maricevic T, et al. Periodontal and prosthetic aspect of biological width, part I: violation of biologic width. Acta Stomatol Croat 2000;34(2):195-7.

[10] Hegde MN, Hegde N, Bhat R. Gummy smile correction: case report. J Laser Opt Photonics 2014;1(1):1-3.

[11] Ardu S, Braut V, Gutemberg D, et al. A long-term laboratory test on staining susceptibility of esthetic composite resin materials. Quintessence Int 2010;41(8):695-702.

[12] Dietschi D. Optimising aesthetics and facilitating clinical application of free-hand bonding using the natural layering concept. Br Dent J 2008;204(4):181-5. 